\title{
A CHARACTERIZATION OF POSITIVE LINEAR MAPS AND CRITERIA OF ENTANGLEMENT FOR QUANTUM STATES
}

\author{
JINCHUAN HOU
}

\begin{abstract}
Let $H$ and $K$ be (finite or infinite dimensional) complex Hilbert spaces. A characterization of positive completely bounded normal linear maps from $\mathcal{B}(H)$ into $\mathcal{B}(K)$ is given, which particularly gives a characterization of positive elementary operators including all positive linear maps between matrix algebras. This characterization is then applied give a representation of quantum channels (operations) between infinite-dimensional systems. A necessary and sufficient criterion of separability is give which shows that a state $\rho$ on $H \otimes K$ is separable if and only if $(\Phi \otimes I) \rho \geq 0$ for all positive finite rank elementary operators $\Phi$. Examples of NCP and indecomposable positive linear maps are given and are used to recognize some entangled states that cannot be recognized by the PPT criterion and the realignment criterion.
\end{abstract}

\section{INTRODUCTION}

Positive linear maps and completely positive linear maps are found to be very important in quantum mechanics, quantum computation and quantum information. In fact they can be used to recognize entangled states, and every quantum channel is represented as a trace preserving completely positive linear map.

In quantum mechanics, a quantum system is associated with a separable complex Hilbert space $H$, i.e., the state space. A quantum state is described as a density operator $\rho \in$ $\mathcal{T}(H) \subseteq \mathcal{B}(H)$ which is positive and has trace 1 , where $\mathcal{B}(H)$ and $\mathcal{T}(H)$ denote the von Neumann algebras of all bounded linear operators and the trace-class of all operators $T$ with $\|T\|_{1}=\operatorname{Tr}\left(\left(T^{\dagger} T\right)^{\frac{1}{2}}\right)<\infty$, respectively. $\rho$ is a pure state if $\rho^{2}=\rho ; \rho$ is a mixed state if $\rho^{2} \neq \rho$. The state space $H$ of a composite quantum system is the tensor product of the state spaces of the component quantum systems $H_{i}$, that is $H=H_{1} \otimes H_{2} \otimes \ldots \otimes H_{k}$. In this paper we are mainly interested in bipartite systems, that is, the case $k=2$. Let $H$ and $K$ be finite dimensional and let $\rho$ be a state acting on $H \otimes K . \rho$ is said to be separable if $\rho$ can be written as

$$
\rho=\sum_{i=1}^{k} p_{i} \rho_{i} \otimes \sigma_{i},
$$

where $\rho_{i}$ and $\sigma_{i}$ are states on $H$ and $K$ respectively, and $p_{i}$ are positive numbers with $\sum_{i=1}^{k} p_{i}=$ 1. Otherwise, $\rho$ is said to be inseparable or entangled (ref. [1, 2]). For the case that at least one of $H$ and $K$ is of infinite dimension, by Werner [3], a state $\rho$ acting on $H \otimes K$ is called

PACS. 03.67.Mn, 03.67.Ud, 03.65.Db.

Key words and phrases. Quantum states, entanglement, positive linear maps.

This work is partially supported by National Natural Science Foundation of China (No. 10771157) and Research Grant to Returned Scholars of Shanxi (2007-38). 
separable if it can be approximated in the trace norm by the states of the form

$$
\sigma=\sum_{i=1}^{n} p_{i} \rho_{i} \otimes \sigma_{i}
$$

where $\rho_{i}$ and $\sigma_{i}$ are states on $H$ and $K$ respectively, and $p_{i}$ are positive numbers with $\sum_{i=1}^{n} p_{i}=$ 1. Otherwise, $\rho$ is called an entangled state.

The quantum entangled states have been used as basic resources in quantum information processing and communication (see $[2,4,45,6,7,6]$ ). Generally, to decide whether or not a state of composite quantum systems is entangled is one of the most challenging task of this field [2]. For the case of $2 \times 2$ or $2 \times 3$ systems, that is, for the case $\operatorname{dim} H=\operatorname{dim} K=2$ or $\operatorname{dim} H=2, \operatorname{dim} K=3$, a state is separable if and only if it is a PPT (Positive Partial Transpose) state [9, 10]. But PPT is only a necessary condition for a state to be separable acting on Hilbert space of higher dimensions. There are PPT states that are entangled. It is known that PPT entangled states belong to the class of bound entangled states [11]. In [12], the realignment criterion for separability in finite dimensional systems was found. It is independent of the PPT criterion and can detect some bound entangled states that cannot be recognized by the PPT criterion. There are several other sufficient criteria for entanglement such as the reduction criteria [13, 14, 15].

A most general approach to study the entanglement of quantum states in finite dimensional systems is based on the notion of entanglement witnesses (see [9]). A Hermitian operator $W$ acting on $H \otimes K$ is said to be an entanglement witness (briefly, EW), if $W$ is not positive and $\operatorname{Tr}(W \sigma) \geq 0$ holds for all separable states $\sigma$. Thus, $\rho$ is entangled if and only if there exists an EW $W$ such that $\operatorname{Tr}(W \rho)<0$ [ ]. This entanglement witness criterion is also valid for infinite dimensional systems. Clearly, constructing entanglement witnesses is a hard task. A recent result in [16] states that every entangled state in a bipartite (finite or infinite dimensional) system can be detected by a witness of the form $c I-F$, where $c$ is a nonnegative number and $F$ is a finite rank self-adjoint operator.

Another general approach to detect entanglement is based on positive maps. It is obvious that if $\rho$ is a state on $H \otimes K$, then for every completely positive (briefly, CP) linear map $\Phi: \mathcal{B}(H) \rightarrow \mathcal{B}(K)$, the operator $(\Phi \otimes I) \rho \in \mathcal{B}(K \otimes K)$ is always positive; if $\rho$ is separable, then for every positive linear map $\Phi: \mathcal{B}(H) \rightarrow \mathcal{B}(K)$, the operator $(\Phi \otimes I) \rho$ is always positive on $K \otimes K$ (or, for every positive linear map $\Phi: \mathcal{B}(K) \rightarrow \mathcal{B}(H)$, the operator $(I \otimes \Phi) \rho$ is always positive on $H \otimes H)$. The converse of the last statement is also true. In [9], it was shown that

Horodeckis' Theorem. [9, Theorem 2] Let H, K be finite dimensional complex Hilbert spaces and $\rho$ be a state acting on $H \otimes K$. Then $\rho$ is separable if and only if for any positive linear map $\Phi: \mathcal{B}(H) \rightarrow \mathcal{B}(K)$, the operator $(\Phi \otimes I) \rho$ is positive on $K \otimes K$.

The positive map criterion and the witness criterion for entanglement are two of few known necessary and sufficient criteria. These two criteria are closely connected by the so-called the Jamiołkowski-Choi isomorphism [9, 17, 18, 19]. Recall that a positive map is said to be decomposable if and only if it is the sum of a CP map and a map which is the transpose of some CP map. It is obvious that a decomposable positive map can not detect any PPT entangled states [20]. 
Let us consider the case that at least one of $H$ and $K$ is of infinite dimension. As every positive linear map $\eta$ between von Neumann algebras is bounded and $\|\eta\|=\|\eta(I)\|$ (see [21, Exercise 10.5.10]), $\rho$ is separable on $H \otimes K$ still implies that, for any completely bounded positive linear map $\Phi: \mathcal{B}(H) \rightarrow \mathcal{B}(K)$, the operator $(\Phi \otimes I) \rho$ is positive on $K \otimes K$. The infinitedimensional version of Horodeckis' Theorem above was obtained by Størmer [22]. Recall that a positive linear map $\Phi: \mathcal{B}(H) \rightarrow \mathcal{B}(K)$ is said to be normal if it is weakly continuous on bounded sets, or equivalently, if it is ultra-weakly continuous (i.e., if $\left\{A_{\alpha}\right\}$ is a bounded net and there is $A \in \mathcal{B}(H)$ such that $\left\langle x\left|A_{\alpha}\right| y\right\rangle$ converges to $\langle y|A| x\rangle$ for any $|x\rangle \in H,|y\rangle \in K$, then $\left\langle x\left|\Phi\left(A_{\alpha}\right)\right| y\right\rangle$ converges to $\langle y|\Phi(A)| x\rangle$ for any $|x\rangle \in H,|y\rangle \in K$. ref. [23, pp.59]).

Størmer's Theorem. [22] Let $H, K$ be Hilbert spaces, $\rho$ be a state acting on $H \otimes K$. Then $\rho$ is separable if and only if for any normal positive linear map $\Phi: \mathcal{B}(H) \rightarrow \mathcal{B}(K)$, the operator $(\Phi \otimes I) \rho$ is positive on $K \otimes K$.

Thus, for a state $\rho$ on $H \otimes K$, if there exists a normal positive map $\Phi: \mathcal{B}(H) \rightarrow \mathcal{B}(K)$ such that $(\Phi \otimes I) \rho$ is not positive or unbounded, then $\rho$ is entangled. In this situation, $\Phi$ can never be completely positive. Therefore, to detect the inseparability of states, the key is to find the normal positive linear maps that are not completely positive. In the case that $\operatorname{dim} H=\operatorname{dim} K=n$, the transpose $A \mapsto A^{T}$ and the map $A \mapsto \operatorname{Tr}(A) I-A$ are well known positive maps that are not completely positive.

Positive linear maps have attracted much attention of physicists working in quantum information science in recent decades because of Horodeckis' positive map criterion. Great efforts have be payed to find as many as possible positive maps that are not $\mathrm{CP}$, and then use them to detect some entangled states [15, 24, 25, 26], for finite dimensional systems. Positive linear maps and completely positive linear maps are also important mathematical topics studied intensively in a general setting of $\mathrm{C}^{*}$-algebras by mathematicians. The completely positive linear maps can be understood quite well. However, the structure of positive linear maps is drastically nontrivial even for the finite dimensional case ([27]-[32]).

Note that every linear map $\Phi$ from $\mathcal{B}(H)$ into $\mathcal{B}(K)$ is an elementary operator if both $H$ and $K$ are finite dimensional, that is, there exist operators $A_{1}, A_{2}, \ldots, A_{k} \in \mathcal{B}(H, K)$ and $B_{1}, B_{2}, \ldots, B_{k} \in \mathcal{B}(K, H)$, such that $\Phi(T)=\sum_{i=1}^{k} A_{i} T B_{i}$ for all $T \in \mathcal{B}(H)$. So, it is also basic important and interesting to find as many as possible characterizations of positive elementary operators and characterizations of completely positive elementary operators, and then, to apply them to get some criteria for the entanglement of states.

A characterization of positive elementary operators was obtained in [31] in terms of contractively locally linear combinations. This is the only known necessary and sufficient condition for an elementary operator to be positive. The purpose of this paper is to give a characterization of positive completely bounded normal maps between $\mathcal{B}(H)$ and $\mathcal{B}(K)$, which including all positive elementary operators. Consequently, we obtain concrete representations of the completely bounded linear maps, positive completely bounded linear maps and completely positive linear maps between the trace-classes $\mathcal{T}(H)$ and $\mathcal{T}(K)$, which allow us to obtain a representation of quantum operations channels (operations) for infinite-dimensional systems. Apply our characterization of positive maps that are not $\mathrm{CP}$, a necessary and a sufficient criterion, that is, the elementary operator criterion of separability is proved. Finally, some positive elementary operators are constructed so that they are not completely positive, even 
indecomposable, and then used to recognize some entangled quantum states that cannot be detected by the PPT criterion and the realignment criterion.

The paper is organized as follows. Section 2 is of mathematics. We show that the set of completely bounded normal linear maps coincides with the set of generalized elementary operators in the setting of separable Hilbert spaces, and give a characterization of positive (completely positive) generalized elementary operators (Lemma 2.1, Theorem 2.5), which improve the main results in [31. Several simple necessary or sufficient conditions to sure that a positive map is not completely positive are also provided. We also show that the noncomplete positivity of a positive elementary operator is essentially determined by its behavior on finite-dimensional subspaces. In Section 3, applying the results in Section 2, some necessary and sufficient conditions for a completely bounded linear map on the trace-class $\mathcal{T}(H)$ to be positive, or to be completely positive are given (see Theorem 3.2). As a corollary, we get a representation of quantum channels (operations) for infinite dimensional systems (Corollary 3.3), which is similar to that for finite dimensional systems. The purpose of Section 4 is to apply the results in Section 2 to get some criteria of entangled quantum states both for finite-dimensional case and infinite-dimensional case and deduce the main result of this paper, i.e., the elementary operator criterion, valid for both finite dimensional systems and infinite dimensional cases. We show that the following statements are equivalent: (1) $\rho$ is separable; (2) $(\Phi \otimes I) \rho \geq 0$ for every positive elementary operator $\Phi$; (3) $(\Phi \otimes I) \rho \geq 0$ for every finite rank positive elementary operator $\Phi$ (Theorem 4.5). Thus, a state $\rho$ is entangled if and only if there exists an elementary operator of the form $\Phi(\cdot)=\sum_{i=1}^{k} C_{i}(\cdot) C_{i}^{\dagger}-\sum_{j=1}^{l} D_{j}(\cdot) D_{j}^{\dagger}$ : $\mathcal{B}(H) \rightarrow \mathcal{B}(K)$, where all $C_{i}$ s and $D_{j}$ s are of finite rank and $\left\{D_{1}, \ldots, D_{l}\right\}$ is a contractive locally linear combination of $\left\{C_{1}, \ldots, C_{k}\right\}$, such that the operator $(\Phi \otimes I) \rho$ is not positive. This criterion improves the Stømer' theorem greatly and is more practical. Section 5 is devoted to illustrating how to apply the results in Sections 2 and 4 to construct positive elementary operators that are not completely positive and even indecomposable (see propositions 5.1-5.2). These maps then are used to distinguish some entangled states that cannot be recognized by PPT criterion as well as the realignment criterion. In Section 6, we give a short conclusion.

Throughout this paper, $H$ and $K$ are separable complex Hilbert spaces that may be of infinite dimension if no specific assumption is made, and $\langle\cdot \mid \cdot\rangle$ stands for the inner product in both of them. $\mathcal{B}(H, K)(\mathcal{B}(H)$ when $K=H)$ is the Banach space of all (bounded linear) operators from $H$ into $K . A \in \mathcal{B}(H)$ is self-adjoint if $A=A^{\dagger}$ ( $A^{\dagger}$ stands for the adjoint operator of $A$ ); and $A$ is positive, denoted by $A \geq 0$, if $A$ is self-adjoint with spectrum falling in the interval $[0, \infty)$ (or equivalently, $\langle\psi \mid A \psi\rangle \geq 0$ for all $|\psi\rangle \in H$ ). For any positive integer $n$, $H^{(n)}$ denotes the direct sum of $n$ copies of $H$. It is clear that every operator $\mathbf{A} \in \mathcal{B}\left(H^{(n)}, K^{(m)}\right)$ can be written in an $n \times m$ operator matrix $\mathbf{A}=\left(A_{i j}\right)_{i, j}$ with $A_{i j} \in \mathcal{B}(H, K), i=1,2, \ldots, m$; $j=1,2, \ldots, n$. Equivalently, $\mathcal{B}\left(H^{(n)}, K^{(m)}\right)$ is often written as $\mathcal{B}(H, K) \otimes \mathcal{M}_{m \times n}(\mathbb{C})$. We will write $\mathbf{A}^{\mathrm{T}}=\left(A_{i j}\right)^{\mathrm{T}}$ for the formal transpose matrix $\left(A_{j i}\right)_{i, j}$ of $\mathbf{A}, \mathbf{A}^{\mathrm{t}}=\left(A_{j i}^{\mathrm{t}}\right)_{i, j}$ for the usual transpose of $\mathbf{A}$, and denote by $A^{(n)}$ the operator matrix $\left(A_{i j}\right) \in \mathcal{B}\left(H^{(n)}, K^{(n)}\right)$ with $A_{i i}=A$ and $A_{i j}=0$ if $i \neq j$. If $\Phi$ is a linear map from $\mathcal{B}(H)$ into $\mathcal{B}(K)$, we can define a linear map $\Phi_{n}: \mathcal{B}\left(H^{(n)}\right) \rightarrow \mathcal{B}\left(K^{(n)}\right)$ by $\Phi_{n}\left(\left(A_{i j}\right)\right)=\left(\Phi\left(A_{i j}\right)\right)$. Recall that $\Phi$ is said to be positive (resp. hermitian-preserving) if $A \in \mathcal{B}(H)$ is positive (resp. self-adjoint) implies that $\Phi(A)$ is positive (resp. self-adjoint). If $\Phi_{n}$ is positive we say $\Phi$ is $n$-positive; if $\Phi_{n}$ 
is positive for every integer $n>0$, we say that $\Phi$ is completely positive. Obviously, $\Phi$ is completely positive $\Rightarrow \Phi$ is positive $\Rightarrow \Phi$ is hermitian-preserving. $\Phi$ is said to be completely bounded if $\|\phi\|_{c b}=\sup _{n}\left\|\Phi_{n}\right\|<\infty$. $\Phi: \mathcal{B}(H) \rightarrow \mathcal{B}(K)$ is called an elementary operator if there are two finite sequences $\left\{A_{i}\right\}_{i=1}^{n} \subset \mathcal{B}(H, K)$ and $\left\{B_{i}\right\}_{i=1}^{n} \subset \mathcal{B}(K, H)$ such that $\Phi(X)=$ $\sum_{i=1}^{n} A_{i} X B_{i}$ for all $X \in \mathcal{B}(H) ; \Phi: \mathcal{B}(H) \rightarrow \mathcal{B}(K)$ is called a generalized elementary operator if there exists sequences $\left\{A_{i}\right\}$ and $\left\{B_{i}\right\}$ satisfying $\left\|\sum_{i} A_{i} A_{i}^{\dagger}\right\|\left\|\sum_{i} B_{i}^{\dagger} B_{i}\right\|<\infty$ such that $\Phi(X)=\sum_{i} A_{i} X B_{i}$ for all $X$. Obviously, the generalized elementary operators are completely bounded and normal.

\section{Characterizing positive Completely Bounded normal maps}

In this section we give a characterization of positive completely bounded normal linear maps from $\mathcal{B}(H)$ into $\mathcal{B}(K)$. To do this, we need a lemma.

Lemma 2.1. Let $H, K$ be separable complex Hilbert spaces and $\Phi: \mathcal{B}(H) \rightarrow \mathcal{B}(K)$ be a linear map. Then $\Phi$ is normal and completely bounded if and only if $\Phi$ is a generalized elementary operator.

Proof. We need only check the "only if" part. Assume that the linear map $\Phi: \mathcal{B}(H) \rightarrow$ $\mathcal{B}(K)$ is completely bounded and normal. It follows that, $\Phi=\Phi_{1}-\Phi_{2}+i\left(\Phi_{3}-\Phi_{4}\right)$ with $\Phi_{i}$ normal and completely positive by Wittstock's decomposition theorem (ref. [32]). As $H$ and $K$ are separable, by Stinespring's Theorem (ref. [32, 33]) and the structural theorem of normal $*$-homomorphisms of $\mathcal{B}(H)$ (ref. [23, pp.61]), for each $k=1,2,3,4$, there exist a countable cardinal number $J_{k}$, an operator $U_{k} \in \mathcal{B}\left(H^{\left(J_{k}\right)}, K\right)$ such that $\Phi_{k}(X)=U_{k} X^{\left(J_{k}\right)} U_{k}^{\dagger}$, where $H^{\left(J_{k}\right)}$ (resp. $X^{\left(J_{k}\right)}$ ) is the direct sum of $J_{k}$-copies of $H$ (resp. of $X$ ). Therefore, there are sequences of operators $\left\{A_{i}\right\}_{i \leq J_{1}},\left\{B_{j}\right\}_{j \leq J_{2}},\left\{C_{s}\right\}_{s \leq J_{3}},\left\{D_{t}\right\}_{t \leq J_{4}} \subset \mathcal{B}(H, K)$, such that

$$
\begin{aligned}
U_{1} & =\left(\begin{array}{lllll}
A_{1} & A_{2} & \cdots & A_{i} & \cdots
\end{array}\right) \\
U_{2} & =\left(\begin{array}{lllll}
B_{1} & B_{2} & \cdots & B_{j} & \cdots
\end{array}\right), \\
U_{3} & =\left(\begin{array}{lllll}
C_{1} & C_{2} & \cdots & C_{s} & \cdots
\end{array}\right), \\
U_{4} & =\left(\begin{array}{lllll}
D_{1} & D_{2} & \cdots & D_{t} & \cdots
\end{array}\right)
\end{aligned}
$$

and

$$
\Phi(X)=\sum_{i \leq J_{1}} A_{i} X A_{i}^{\dagger}-\sum_{j \leq J_{2}} B_{j} X B_{j}^{\dagger}+i \sum_{s \leq J_{3}} C_{s} X C_{s}^{\dagger}-i \sum_{t \leq J_{4}} D_{t} X D_{t}^{\dagger}
$$

for every $X \in \mathcal{B}(H)$. Now it is clear that

$$
\left\|\sum_{i \leq J_{1}} A_{i} A_{i}^{\dagger}+\sum_{j \leq J_{2}} B_{i} B_{i}^{\dagger}+\sum_{s \leq J_{3}} C_{s} C_{s}^{\dagger}+\sum_{\leq J_{4}} t D_{t} D_{t}^{\dagger}\right\| \leq \sum_{k=1}^{4}\left\|U_{k}\right\|^{2}<\infty,
$$

and so, $\Phi$ is a generalized elementary operator.

By Lemma 2.1, the question of characterizing positive completely bounded normal linear maps between $\mathcal{B}(H)$ and $\mathcal{B}(K)$ is equivalent to the question of characterizing positive generalized elementary operators.

As a special class of generalized elementary operators, the global structures of hermitianpreserving and completely positive elementary operators are quite clear. In fact, for generalized elementary operators, by the proof of Lemma 2.1, we have the following result. 
Corollary 2.2. Let $H, K$ be Hilbert spaces and $\Phi$ be a generalized elementary operator from $\mathcal{B}(H)$ into $\mathcal{B}(K)$. Then

(i) $\Phi$ is hermitian-preserving if and only if there are sequences $\left\{A_{i}\right\},\left\{C_{j}\right\} \subset \mathcal{B}(H, K)$ with $\left\|\sum_{i=1}^{\infty} A_{i} A_{i}^{\dagger}\right\|<\infty$ and $\left\|\sum_{j=1}^{\infty} C_{j} C_{j}^{\dagger}\right\|<\infty$ such that

$$
\Phi(X)=\sum_{i=1}^{\infty} A_{i} X A_{i}^{\dagger}-\sum_{j=1}^{\infty} C_{j} X C_{j}^{\dagger}
$$

for every $X \in \mathcal{B}(H)$;

(ii) $\Phi$ is completely positive if and only if there exists a sequence $\left\{A_{i}\right\} \subset \mathcal{B}(H, K)$ with $\left\|\sum_{i=1}^{\infty} A_{i} A_{i}^{\dagger}\right\|<\infty$ such that

$$
\Phi(X)=\sum_{i=1}^{\infty} A_{i} X A_{i}^{\dagger}
$$

for every $X \in \mathcal{B}(H)$.

If both $H$ and $K$ are finite-dimensional, Theorem 2.1(i) and (ii) were established by DePillis [30] and Choi [27], respectively. For the elementary operator case, see [34] and [35].

For a sequence $\mathbf{A}=\left(\begin{array}{lllll}A_{1} & A_{2} & \cdots & A_{i} & \cdots\end{array}\right)$, we will denote by $\mathbf{A}^{T}$ the formal transpose of $\mathbf{A}$ and $\mathbf{A}^{\dagger}$ the usual adjoint operator of $\mathbf{A}$, that is

$$
\mathbf{A}^{T}=\left(\begin{array}{c}
A_{1} \\
A_{2} \\
\vdots \\
A_{i} \\
\vdots
\end{array}\right) \quad \text { and } \quad \mathbf{A}^{\dagger}=\left(\begin{array}{c}
A_{1}^{\dagger} \\
A_{2}^{\dagger} \\
\vdots \\
A_{i}^{\dagger} \\
\vdots
\end{array}\right)
$$

We will also denote by $\mathcal{B}_{1}(H, K)$ the closed unit ball of $\mathcal{B}(H, K)$.

The next lemma is the key lemma which is a generalization of [31, Lemma 2.2], where more conditions $\left\|\sum_{i=1}^{\infty} A_{i}^{\dagger} A_{i}\right\|<\infty$ and $\left\|\sum_{j=1}^{\infty} C_{j}^{\dagger} C_{j}\right\|<\infty$ are assumed. Note that, the conditions $\left\|\sum_{i=1}^{\infty} A_{i} A_{i}^{\dagger}\right\|<\infty$ and $\left\|\sum_{i=1}^{\infty} A_{i}^{\dagger} A_{i}\right\|<\infty$ are not equivalent in general. For instance, let $H=\oplus_{i=1}^{\infty} H_{i}$ with each $H_{i}$ is of infinite dimension. Let $V_{i} \in \mathcal{B}(H)$ be the isometry with range $H_{i}$. Then $V_{i}^{\dagger} V_{i}=I$ and $V_{i} V_{i}^{\dagger}=P_{i}$, where $P_{i}$ is the projection from $H$ onto $H_{i}$. Thus $\left\|\sum_{i=1}^{\infty} V_{i} V_{i}^{\dagger}\right\|=\left\|\sum_{i=1}^{\infty} P_{i}\right\|=\|I\|=1$ as $P_{i} P_{j}=0$ whenever $i \neq j$, but $\left\|\sum_{i=1}^{\infty} V_{i}^{\dagger} V_{i}\right\|=\infty$.

Lemma 2.3. Let $\left\{A_{i}\right\}_{i=1}^{\infty}$ and $\left\{C_{j}\right\}_{j=1}^{\infty} \subset \mathcal{B}(H, K)$ with $\left\|\sum_{i=1}^{\infty} A_{i} A_{i}^{\dagger}\right\|<\infty$ and $\|$ $\sum_{j=1}^{\infty} C_{j} C_{j}^{\dagger} \|<\infty$. Then the following statements are equivalent:

(i) $\sum_{i=1}^{\infty} A_{i} P A_{i}^{\dagger} \geq \sum_{j=1}^{\infty} C_{j} P C_{j}^{\dagger}$ for all positive operators $P \in \mathcal{B}(H)$.

(ii) $\sum_{i=1}^{\infty} A_{i} P A_{i}^{\dagger} \geq \sum_{j=1}^{\infty} C_{j} P C_{j}^{\dagger}$ for all rank-one projections $P \in \mathcal{B}(H)$.

(iii) There exists a map $\Omega: H \rightarrow \mathcal{B}_{1}\left(l_{2}\right)$ such that

$$
\mathbf{C}^{T}|\psi\rangle=\Omega(|\psi\rangle) \mathbf{A}^{T}|\psi\rangle \quad \text { for every }|\psi\rangle \in H .
$$

Proof. (i) $\Rightarrow($ ii $) \Rightarrow$ (iii) were done in the proof of [31, Lemma 2.2].

(iii) $\Rightarrow$ (ii). Assume (iii). For any unit vector $|\psi\rangle \in H$, denote $P=|\psi\rangle\langle\psi|$ and the contractive matrix $\Omega(|\psi\rangle)=\Omega=\left(\omega_{i j}\right)$. As $\mathbf{C}^{T}|\psi\rangle=\Omega(|\psi\rangle) \mathbf{A}^{T}|\psi\rangle$, we have $C_{i}|\psi\rangle=\sum_{j=1}^{\infty} \omega_{i j} A_{j}|\psi\rangle$ for 
each $i$. Thus,

$$
\begin{aligned}
\mathbf{C} P & =\left(C_{1} P, C_{2} P, \ldots, C_{j} P, \ldots\right) \\
& =\left(\sum_{j=1}^{\infty} \omega_{1 j} A_{j} P, \sum_{j=1}^{\infty} \omega_{2 j} A_{j} P, \ldots, \sum_{j=1}^{\infty} \omega_{i j} A_{j} P, \ldots\right) \\
& =\left(A_{1} P, A_{2} P, \ldots, A_{j} P, \ldots\right) \Omega^{T} \\
& =\left(A_{1} P, A_{2} P, \ldots, A_{j} P, \ldots\right)\left(w_{i j} I\right)^{T}=\mathbf{A} P\left(w_{i j} I\right)^{T} .
\end{aligned}
$$

It follows that

$$
\sum_{i=1}^{\infty} C_{i} P C_{i}^{\dagger}=\mathbf{C} P \mathbf{C}^{\dagger}=\mathbf{A} P\left(\omega_{i j} I\right)^{T}\left(\left(\omega_{i j} I\right)^{T}\right)^{\dagger} P \mathbf{A}^{\dagger} \leq \mathbf{A} P \mathbf{A}^{\dagger}=\sum_{j=1}^{\infty} A_{j} P A_{j}^{\dagger}
$$

because of $0 \leq\left(\omega_{i j} I\right)^{T}\left(\left(\omega_{i j} I\right)^{T}\right)^{\dagger} \leq I$.

(ii) $\Rightarrow$ (i). Let $\Delta(X)=\sum_{j=1}^{\infty} A_{j} X A_{j}^{\dagger}-\sum_{i=1}^{\infty} C_{i} X C_{i}^{\dagger}=\mathbf{A} X^{(\infty)} \mathbf{A}^{\dagger}-\mathbf{C} X^{(\infty)} \mathbf{C}^{\dagger}$ for each $X \in \mathcal{B}(H)$. Since $\|\mathbf{A}\|=\left\|\mathbf{A} \mathbf{A}^{\dagger}\right\|^{\frac{1}{2}}=\left(\left\|\sum_{j=1}^{\infty} A_{j} A_{j}^{\dagger}\right\|\right)^{\frac{1}{2}}<\infty$ and $\|\mathbf{C}\|=\left\|\mathbf{C C}^{\dagger}\right\|^{\frac{1}{2}}=$ $\left(\left\|\sum_{i=1}^{\infty} C_{i} C_{i}^{\dagger}\right\|\right)^{\frac{1}{2}}<\infty$, we see that $\Delta$ is normal. The condition (ii) implies that $\Delta(P)$ is positive for every finite rank positive operator $P$. For any positive operator $X \in \mathcal{B}(H)$, by spectral theorem, there exists a net $P_{\lambda}$ of finite-rank positive operators such that $\left\|P_{\lambda}\right\| \leq\|X\|$ and wk $-\lim _{\lambda} P_{\lambda}=X$. Hence $\Delta(X)=\mathrm{wk}-\lim _{\lambda} \Delta\left(P_{\lambda}\right)$ is positive and (i) is true.

The next Lemma is a generalization of the main result [31, Theorem 2.4].

Lemma 2.4. Let $H, K$ be complex Hilbert spaces and $\left\{A_{i}\right\}_{i=1}^{\infty},\left\{C_{j}\right\}_{j=1}^{\infty} \subset \mathcal{B}(H, K)$ with $\left\|\sum_{i=1}^{\infty} A_{i} A_{i}^{\dagger}\right\|<\infty$ and $\left\|\sum_{j=1}^{\infty} C_{j} C_{j}^{\dagger}\right\|<\infty$. Let $\Phi: \mathcal{B}(H) \rightarrow \mathcal{B}(K)$ be a linear map defined by

$$
\Phi(X)=\sum_{i=1}^{\infty} A_{i} X A_{i}^{\dagger}-\sum_{j=1}^{\infty} C_{j} X C_{j}^{\dagger}
$$

for every $X \in \mathcal{B}(H)$. Then

(i) $\Phi$ is positive if and only if there exists a map $\Omega:|\psi\rangle \in H \mapsto \Omega(|\psi\rangle)=\left(\omega_{j i}(|\psi\rangle)\right)_{j, i} \in$ $\mathcal{B}_{1}\left(l_{2}\right)$ such that

$$
\mathbf{C}^{T}|\psi\rangle=\Omega(|\psi\rangle) \mathbf{A}^{T}|\psi\rangle
$$

for every $|\psi\rangle \in H$.

(ii) $\Phi$ is completely positive if and only if there exists a contractive matrix $\Omega=\left(\omega_{j i}\right)_{j, i} \in$ $\mathcal{B}\left(l_{2}\right)$ such that

$$
\mathbf{C}^{T}=\Omega \mathbf{A}^{T},
$$

and in turn, if and only if there exists a sequence $\left\{D_{i}\right\}_{i=1}^{\infty} \subset \mathcal{B}(H, K)$ such that

$$
\Phi(X)=\sum_{i=1}^{\infty} D_{i} X D_{i}^{\dagger}
$$

holds for all $X \in \mathcal{B}(H)$.

Here $\mathbf{A}=\left(A_{1}, A_{2}, \ldots, A_{n}, \ldots\right)$ and $\mathbf{C}=\left(C_{1}, C_{2}, \ldots, C_{n}, \ldots\right)$.

Proof. By Lemma 2.3, [31, Theorem 2.4] and its proof, we know that the lemma holds except the conclusion that $\Phi$ is completely positive if and only if there exists a sequence $\left\{D_{i}\right\}_{i=1}^{\infty} \subset \mathcal{B}(H, K)$ such that

$$
\Phi(X)=\sum_{i=1}^{\infty} D_{i} X D_{i}^{\dagger}
$$

for every $X \in \mathcal{B}(H)$. But this is true by Corollary 2.2. 
Combine Lemma 2.1 and Lemma 2.4, one gets the main result of this section immediately.

Theorem 2.5. Let $H, K$ be separable complex Hilbert spaces and $\Phi: \mathcal{B}(H) \rightarrow \mathcal{B}(K)$ be a completely bounded normal linear map. Then

(1) $\Phi$ is positive if and only if there exist $\left\{A_{i}\right\}_{i=1}^{\infty},\left\{C_{j}\right\}_{j=1}^{\infty} \subset \mathcal{B}(H, K)$ with $\left\|\sum_{i=1}^{\infty} A_{i} A_{i}^{\dagger}\right\|<$ $\infty$ and $\left\|\sum_{j=1}^{\infty} C_{j} C_{j}^{\dagger}\right\|<\infty$, and a map $\Omega:|\psi\rangle \in H \mapsto \Omega(|\psi\rangle)=\left(\omega_{j i}(|\psi\rangle)\right)_{j, i} \in \mathcal{B}_{1}\left(l_{2}\right)$ satisfying

$$
\mathbf{C}^{T}|\psi\rangle=\Omega(|\psi\rangle) \mathbf{A}^{T}|\psi\rangle
$$

for every $|\psi\rangle \in H$, such that

$$
\Phi(X)=\sum_{i=1}^{\infty} A_{i} X A_{i}^{\dagger}-\sum_{j=1}^{\infty} C_{j} X C_{j}^{\dagger}
$$

holds for every $X \in \mathcal{B}(H)$.

(2) $\Phi$ is completely positive if and only if there exists a sequence $\left\{D_{i}\right\}_{i=1}^{\infty} \subset \mathcal{B}(H, K)$ with $\left\|\sum_{i=1}^{\infty} D_{i} D_{i}^{\dagger}\right\|<\infty$ such that

$$
\Phi(X)=\sum_{i=1}^{\infty} D_{i} X D_{i}^{\dagger}
$$

holds for all $X \in \mathcal{B}(H)$.

Here $\mathbf{A}=\left(A_{1}, A_{2}, \ldots, A_{n}, \ldots\right)$ and $\mathbf{C}=\left(C_{1}, C_{2}, \ldots, C_{n}, \ldots\right)$.

What does Theorem 2.5 mean? To understand Theorem 2.5 better, let us recall some notions from [31. Let $l, k \in \mathbb{N}$ (the set of all natural numbers), and let $A_{1}, \cdots, A_{k}$, and $C_{1}, \cdots, C_{l} \in \mathcal{B}(H, K)$. If, for each $|\psi\rangle \in H$, there exists an $l \times k$ complex matrix $\left(\alpha_{i j}(|\psi\rangle)\right)$ (depending on $|\psi\rangle$ ) such that

$$
C_{i}|\psi\rangle=\sum_{j=1}^{k} \alpha_{i j}(|\psi\rangle)\left|A_{j} \psi\right\rangle, \quad i=1,2, \cdots, l,
$$

we say that $\left(C_{1}, \cdots, C_{l}\right)$ is a locally linear combination of $\left(A_{1}, \cdots, A_{k}\right),\left(\alpha_{i j}(|\psi\rangle)\right)$ is called a local coefficient matrix at $|\psi\rangle$. Furthermore, if a local coefficient matrix $\left(\alpha_{i j}(|\psi\rangle)\right)$ can be chosen for every $|\psi\rangle \in H^{(n)}$ so that the operator norm $\|\left(\alpha_{i j}(|\psi\rangle)\right) \| \leq 1$, we say that $\left(C_{1}, \cdots, C_{l}\right)$ is a contractive locally linear combination of $\left(A_{1}, \cdots, A_{k}\right)$; if there is a matrix $\left(\alpha_{i j}\right)$ with $\|\left(\alpha_{i j} \| \leq 1\right.$ such that $C_{i}=\sum_{j=1}^{k} \alpha_{i j} A_{j}$ for all $i$, we say that $\left(C_{1}, \cdots, C_{l}\right)$ is a contractive linear combination of $\left(A_{1}, \cdots, A_{k}\right)$ with coefficient matrix $\left(\alpha_{i j}\right)$. Sometimes we also write $\left\{A_{i}\right\}_{i=1}^{k}$ for $\left(A_{1}, \cdots, A_{k}\right)$. These notions can be generalized to the case that there are infinite many $A_{k} \mathrm{~s}$ or $C_{k} \mathrm{~s}$. For instance, if, for every $|\psi\rangle \in H$, there are scalars $\alpha_{k}(|\psi\rangle)$ such that $C|\psi\rangle=\sum_{k=1}^{\infty} \alpha_{k}(|\psi\rangle) A_{k}|\psi\rangle$ and $\sum_{k=1}^{\infty} \mid\left.\alpha_{k}(|\psi\rangle)\right|^{2} \leq 1$, we will say that $C$ is a generalized contractive locally linear combination of $\left\{A_{k}\right\}_{k=1}^{\infty}$.

Thus Theorem 2.5 may be restated as follows: A completely bounded normal linear map $\Phi: \mathcal{B}(H) \rightarrow \mathcal{B}(K)$ is positive but not completely positive (briefly, NCP) if and only if it has the form $\Phi(X)=\sum_{i=1}^{\infty} A_{i} X A_{i}^{\dagger}-\sum_{j=1}^{\infty} C_{j} X C_{j}^{\dagger}$ for all $X$, where $\left\{C_{j}\right\}$ is a generalized contractive locally linear combination of $\left\{A_{i}\right\}$ but $\left\{C_{j}\right\}$ is not a generalized contractive linear combination of $\left\{A_{i}\right\}$. This characterization is much helpful in some sense to understand the differences of completely positive normal linear maps, positive completely bounded normal linear maps and hermitian completely bounded normal linear maps. 
By Theorem 2.5, one gets immediately a global structure theorem for positive elementary operators in terms of local linear combination that was established in [31]. For $\mathcal{L} \subset \mathcal{B}(H, K)$, we'll denote by $[\mathcal{L}]$ the linear span of $\mathcal{L}$.

Corollary 2.6. Let $\Phi=\sum_{i=1}^{n} A_{i}(\cdot) B_{i}$ be an elementary operator from $\mathcal{B}(H)$ into $\mathcal{B}(K)$. Then $\Phi$ is positive if and only if there exist $C_{1}, \cdots, C_{k}$ and $D_{1}, \cdots, D_{l}$ in $\left[A_{1}, \cdots, A_{n}\right]$ with $k+l \leq n$ such that $\left(D_{1}, \cdots, D_{l}\right)$ is an contractive locally linear combination of $\left(C_{1}, \cdots, C_{k}\right)$ and

$$
\Phi=\sum_{i=1}^{k} C_{i}(\cdot) C_{i}^{\dagger}-\sum_{j=1}^{l} D_{j}(\cdot) D_{j}^{\dagger} .
$$

Furthermore, $\Phi$ in Eq.(2.1) is completely positive if and only if $\left(D_{1}, \cdots, D_{l}\right)$ is a linear combination of $\left(C_{1}, \cdots, C_{k}\right)$ with a contractive coefficient matrix, and in turn, if and only if there exist $E_{1}, E_{2}, \ldots, E_{r}$ with $r \leq k$ such that

$$
\Phi=\sum_{i=1}^{r} E_{i}(\cdot) E_{i}^{\dagger} .
$$

Since every linear map between matrix algebras is an elementary operator, by Corollary 2.6 we get a characterization of positive maps that is not CP for finite dimensional case.

Corollary 2.7. Let $H$ and $K$ be finite dimensional complex Hilbert spaces and let $\Phi$ : $\mathcal{B}(H) \rightarrow \mathcal{B}(K)$ be a linear map. Then $\Phi$ is positive but not completely positive if and only if there exist $C_{1}, \cdots, C_{k}, D_{1}, \cdots, D_{l} \in \mathcal{B}(H, K)$ such that $\Phi(X)=\sum_{i=1}^{k} C_{i} X C_{i}^{\dagger}-\sum_{j=1}^{l} D_{j} X D_{j}^{\dagger}$ for all $X \in \mathcal{B}(H)$, and $\left\{D_{j}\right\}_{j=1}^{l}$ is a contractive locally linear combination but not a contractive linear combination of $\left\{C_{i}\right\}_{i=1}^{k}$.

It is interesting to observe from the discussion above that, for elementary operators, the question when positivity ensures complete positivity may be reduced to the question when contractive locally linear combination implies linear combination. This connection allows us to look more deeply into the relationship and the difference between positivity and complete positivity, and obtain some simple criteria to check whether a positive elementary operator is completely positive or not. This is important especially when we construct positive maps and apply them to recognize entanglement.

If $\mathcal{L} \subset \mathcal{B}(H, K)$, we will denote by $\mathcal{L}_{F}$ the subset of all finite-rank operators in $\mathcal{L}$.

The Corollaries 2.8 and 2.9 below can be found in [31. We list them here for completeness and for reader's convenience.

Corollary 2.8. Assume that $\Phi=\sum_{i=1}^{k} A_{i}(\cdot) A_{i}^{*}-\sum_{j=1}^{l} B_{j}(\cdot) B_{j}^{*}: \mathcal{B}(H) \rightarrow \mathcal{B}(K)$ is a positive elementary operator. If any one of the following conditions holds, then $\Phi$ is completely positive:

(i) $k \leq 2$.

(ii) $\operatorname{dim}\left[A_{1}, \cdots, A_{k}\right]_{F} \leq 2$.

(iii) There exists a vector $|\psi\rangle \in H$ such that $\left\{\left|A_{i} \psi\right\rangle\right\}_{i=1}^{k}$ is linearly independent.

(iv) $\Phi$ is $\left[\frac{k+1}{2}\right]$-positive, where $[t]$ stands for the integer part of real number $t$.

Corollary 2.9. Assume that $\Phi=\sum_{i=1}^{k} A_{i}(\cdot) A_{i}^{*}-\sum_{j=1}^{l} B_{j}(\cdot) B_{j}^{*}: \mathcal{B}(H) \rightarrow \mathcal{B}(K)$ is a positive elementary operator. If $\Phi$ is not completely positive, then

(i) $k \geq 3$,

(ii) $\operatorname{dim}\left[A_{1}, \cdots, A_{k}\right]_{F} \geq 3$, 
(iii) $B_{j}, j=1,2, \ldots, l$, is a finite-rank perturbation of some combination of $\left\{A_{i}\right\}_{i=1}^{k}$.

(iv) $\Phi_{\left[\frac{k+1}{2}\right]}$ is not positive.

Corollary 2.10. Assume that $\Phi=\sum_{i=1}^{k} A_{i}(\cdot) A_{i}^{*}-\sum_{j=1}^{l} B_{j}(\cdot) B_{j}^{*}: \mathcal{B}(H) \rightarrow \mathcal{B}(K)$ is an elementary operator. If there exists some $j$ such that $B_{j}$ is not a contractive linear combination of $\left\{A_{i}\right\}_{i=1}^{k}$, then $\Phi$ is not completely positive.

The following result reveals that the non-complete positivity of a positive elementary operator is essentially determined by its behavior on finite-dimensional subspaces. So, to construct a NCP positive elementary operator, it is enough to consider the question in finite-dimensional cases.

Theorem 2.11. Assume that $\Phi: \mathcal{B}(H) \rightarrow \mathcal{B}(K)$ is a positive elementary operator. Then $\Phi$ is NCP if and only if there exist finite-rank projections $P$ and $Q$ acting on $H$ and $K$, respectively, such that the positive elementary operator $\Delta: \mathcal{B}(P H) \rightarrow \mathcal{B}(Q K)$ defined by $\Delta(X)=\left.Q \Phi(P X P) Q\right|_{Q K}$ is non-completely positive. In addition, $P$ and $Q$ may be taken so that $\Delta^{\prime}: \mathcal{B}(\operatorname{ker} P) \rightarrow \mathcal{B}(\operatorname{ker} Q)$ defined by $\Delta^{\prime}(Y)=(I-Q) \Phi\left(\left.((I-P) Y(I-P))(I-Q)\right|_{\operatorname{ker} Q}\right.$ is completely positive.

Proof. Clearly, if $\Phi: \mathcal{B}(H) \rightarrow \mathcal{B}(K)$ is a positive linear map and $P \in \mathcal{B}(H), Q \in \mathcal{B}(K)$ are projections, then $\Delta: \mathcal{B}(P H) \rightarrow \mathcal{B}(Q K)$ defined by $\Delta(X)=Q \Phi(P X P) Q$ is positive and $\Delta$ is NCP implies that $\Phi$ is NCP.

Assume that $\Phi$ is a positive elementary operator, writing $\Phi=\sum_{i=1}^{k} A_{i}(\cdot) A_{i}^{*}-\sum_{j=1}^{l} B_{j}(\cdot) B_{j}^{*}$ with $\left\{A_{1}, \ldots, A_{k}, B_{1}, \ldots, B_{l}\right\}$ linearly independent. By Corollary 2.9 (ii)-(iii), if $\Phi$ is NCP, then the linear subspace spanned by $\left\{A_{i}\right\}_{i=1}^{k}$ has many finite rank operators and there exists $C_{j} \in\left[A_{1}, A_{2}, \ldots, A_{k}\right]$ and finite rank operators $F_{j} \notin\left[A_{1}, \ldots, A_{k}\right]$ such that $B_{j}=C_{j}+F_{j}$. Let $P_{0}$ be the projection with range the finite dimensional linear subspace spanned by all the ranges of $\left\{E^{\dagger}: E \in\left[A_{1}, \ldots, A_{k}\right]_{\mathcal{F}}\right\}$ and the ranges of $\left\{F_{j}^{\dagger}\right\}_{j=1}^{l}$; and $Q_{0}$ the projection with range the finite dimensional linear subspace spanned by all the ranges of $\left\{E: E \in\left[A_{1}, \ldots, A_{k}\right]_{\mathcal{F}}\right\}$ and the ranges of $\left\{F_{j}\right\}_{j=1}^{l}$. It is easily checked that there exist some finite rank projections $P \geq P_{0}$ and $Q \geq Q_{0}$ such that $Q B_{j} P \notin\left[Q A_{1} P, \ldots, Q A_{k} P\right]$ since $B_{j} \notin\left[A_{1}, \ldots, A_{k}\right]$. Pick such $P$ and $Q$. Let $S_{i}=\left.Q A_{i}\right|_{P H}, i=1,2, \ldots, k$, and $T_{j}=\left.Q B_{j}\right|_{P H}, j=1,2, \ldots, l$. Let $\Delta: \mathcal{B}(P H) \rightarrow \mathcal{B}(Q K)$ be the map defined by $\Delta(X)=\sum_{i=1}^{k} S_{i} X S_{i}^{*}-\sum_{j=1}^{l} T_{j} X T_{j}^{*}=\left.Q \Phi(P X P) Q\right|_{Q K}$. Then $\Delta$ is positive. By the choice of $P$ and $Q, T_{j}$ is not in $\left[S_{1}, \ldots, S_{k}\right]$ for some $j$. Hence, $\Delta$ is not completely positive by Corollary 2.9. Since $\left[(I-Q) A_{1}(I-P), \ldots,(I-Q) A_{k}(I-P)\right]_{\mathcal{F}}=\{0\}$, by Corollary, $\Delta^{\prime}$ is completely positive.

To conclude this section, we give a simple example illustrating that how to use the results in this section to judge whether or not a map is positive, completely positive.

Example 2.12. Assume that $\operatorname{dim} H=n$ and $\{|i\rangle\}_{i=1}^{n}$ is an orthonormal basis. Denote $E_{i j}=|i\rangle\langle j|$. For a given positive number $t$, let $\Delta_{t}: \mathcal{B}(H) \rightarrow \mathcal{B}(H)$ be a linear map defined by

$$
\Delta_{t}(X)=t \sum_{i=1}^{n} E_{i i} X E_{i i}-X
$$

for any $X \in \mathcal{B}(H)$. Then $\Delta_{t}$ is positive if and only if it is completely positive, and in turn, if and only if $t \geq n$.

In fact, let $A_{i}=\sqrt{t} E_{i i}$, then $\Delta_{t}(X)=\sum_{i=1}^{n} A_{i} X A_{i}^{\dagger}-I X I^{\dagger}$. It is clear that $I$ is a linear combination of $A_{1}, \cdots, A_{n}$, i.e., $I=\sum_{i=1}^{n} \frac{1}{\sqrt{t}} A_{i}$. Then the sum of the square of the coefficients 
is $\sum_{i}\left(\frac{1}{\sqrt{t}}\right)^{2}=\frac{n}{t}$, and hence $\Delta_{t}$ is completely positive if and only if $t \geq n$ by Corollary 2.6. If $t<n$, then it is obvious that $I$ is not a contractive locally linear combination of $A_{1}, \cdots, A_{n}$, and hence $\Delta_{t}$ is not positive.

\section{Characterizing Quantum Channels For infinite Dimensional Systems}

It is known that, for finite-dimensional quantum systems, a quantum channel (operation) $\mathcal{E}$ is a trace-preserving (trace-nonincreasing) completely positive linear map between associated matrix algebras. Thus, by a result due to Choi [27], $\mathcal{E}$ is an elementary operator of the form $\mathcal{E}(\cdot)=\sum_{i=1}^{n} A_{i}(\cdot) A_{i}^{\dagger}$, where $\sum_{i=1}^{n} A_{i}^{\dagger} A_{i}=I\left(\sum_{i=1}^{n} A_{i}^{\dagger} A_{i} \leq I\right)$. Using the discussion in Section 2 , one can characterize the completely bounded linear maps, positive completely bounded linear maps and completely positive linear maps between the trace-classes. This allow us to obtain a similar representation of quantum operations for infinite-dimensional systems. Firstly we recall some notions. For $A \in \mathcal{B}(H)$, denote $|A|=\left(A^{\dagger} A\right)^{\frac{1}{2}}$. Recall that the trace class $\mathcal{T}(H)=\left\{T:\|T\|_{1}=\operatorname{Tr}(|T|)<\infty\right\}$, which is a ideal of $\mathcal{B}(H)$. Furthermore, $\mathcal{T}(H)$ is a Banach space with the trace norm $\|\cdot\|_{1}$. The dual space of $\mathcal{T}(H)$ is $\mathcal{T}(H)^{*}=\mathcal{B}(H)$ and every bounded linear functional is of the form $T \mapsto \operatorname{Tr}(A T)$, where $A \in \mathcal{B}(H)$.

Lemma 3.1. Let $H, K$ be separable complex Hilbert spaces and $\mathcal{T}(H), \mathcal{T}(K)$ be the trace classes on $H, K$ respectively. Then, a linear map $\Delta: \mathcal{T}(H) \rightarrow \mathcal{T}(K)$ is completely bounded if and only if there exists operator sequences $\left\{A_{i}\right\}_{i} \subset \mathcal{B}(H, K)$ and $\left\{B_{i}\right\}_{i} \subset \mathcal{B}(K, H)$ satisfying $\left\|\sum_{i} A_{i}^{\dagger} A_{i}\right\|<\infty$, and $\left\|\sum_{i} B_{i} B_{i}^{\dagger}\right\|<\infty$ such that

$$
\Delta(T)=\sum_{i} A_{i} T B_{i}
$$

for all $T \in \mathcal{T}(H)$.

Proof. If $\Delta$ has the form stated in the theorem, it is obvious that, for any $X \in \mathcal{B}(K)$,

$$
\operatorname{Tr}\left(\sum_{i} A_{i} T B_{i} X\right)=\sum_{i} \operatorname{Tr}\left(A_{i} T B_{i} X\right)=\sum_{i} \operatorname{Tr}\left(T B_{i} X A_{i}\right)=\operatorname{Tr}\left(\sum_{i} T B_{i} X A_{i}\right)
$$

holds for all $T \in \mathcal{T}(H)$, so $\Delta^{*}(X)=\sum_{i} B_{i} X A_{i} \in \mathcal{B}(H)$. As $\left\|\sum_{i} A_{i}^{\dagger} A_{i}\right\|<\infty$, and $\|$ $\sum_{i} B_{i} B_{i}^{\dagger} \|<\infty, \Delta^{*}$ is completely bounded with $\left\|\Delta^{*}\right\|_{\mathrm{cb}} \leq\left\|\left(\sum_{i} A_{i}^{\dagger} A_{i}\right)^{\frac{1}{2}}\right\| \cdot\left\|\left(\sum_{i} B_{i} B_{i}^{\dagger}\right)^{\frac{1}{2}}\right\|$. But $\left\|\Delta_{n}\right\|=\left\|\Delta_{n}^{*}\right\|$ (ref. [36, Proposition 3.2.2]), so, $\Delta$ is completely bounded.

Conversely, assume that $\Delta: \mathcal{T}(H) \rightarrow \mathcal{T}(K)$ is a completely bounded linear map; then $\Delta^{*}: \mathcal{B}(K) \rightarrow \mathcal{B}(H)$ is a completely bounded normal linear map. By Lemma 2.1, $\Delta^{*}$ is a generalized elementary operator. So there exists operator sequences $\left\{A_{i}\right\}_{i} \subset \mathcal{B}(H, K)$ and $\left\{B_{i}\right\}_{i} \subset \mathcal{B}(K, H)$ satisfying $\left\|\sum_{i} A_{i}^{\dagger} A_{i}\right\|<\infty$, and $\left\|\sum_{i} B_{i} B_{i}^{\dagger}\right\|<\infty$ such that $\Delta^{*}(X)=$ $\sum_{i} B_{i} X A_{i}$ holds for all $X \in \mathcal{B}(K, H)$. Now, it is clear that $\Delta(T)=\sum_{i} A_{i} T B_{i}$ holds for all $T \in \mathcal{B}(K, H)$, completing the proof.

By Lemma 3.1 and Theorem 2.5 the following results are immediate.

Theorem 3.2. Let $H, K$ be separable complex Hilbert spaces and $\mathcal{T}(H), \mathcal{T}(K)$ be the trace classes on $H, K$ respectively. Let $\Delta: \mathcal{T}(H) \rightarrow \mathcal{T}(K)$ be a linear map. Then

(i) $\Delta$ is positive and completely bounded if and only if there exists operator sequences $\left\{A_{i}\right\}_{i} \subset \mathcal{B}(H, K)$ and $\left\{B_{i}\right\}_{i} \subset \mathcal{B}(H, K)$ with $\left\|\sum_{i} A_{i}^{\dagger} A_{i}\right\|<\infty$ and $\left\|\sum_{i} B_{i}^{\dagger} B_{i}\right\|<\infty$, 
and a map $\Omega: H \rightarrow \mathcal{B}_{1}\left(l_{2}\right)$ such that $\mathbf{B}^{\dagger}|\psi\rangle=\Omega(|\psi\rangle) \mathbf{A}^{\dagger}|\psi\rangle$ for every $|\psi\rangle \in H$ and

$$
\Delta(T)=\sum_{i} A_{i} T A_{i}^{\dagger}-\sum_{i} B_{j} T B_{j}^{\dagger}
$$

for all $T \in \mathcal{T}(H)$.

(ii) $\Delta$ is completely positive if and only if there exists operator sequences $\left\{A_{i}\right\}_{i} \subset \mathcal{B}(H, K)$ with $\left\|\sum_{i} A_{i}^{\dagger} A_{i}\right\|<\infty$ such that

$$
\Delta(T)=\sum_{i} A_{i} T A_{i}^{\dagger}
$$

for all $T \in \mathcal{T}(H)$.

Corollary 3.3. Every quantum channel (operation) $\mathcal{E}$ between two infinite-dimensional systems respectively associated with Hilbert spaces $H$ and $K$ has the form

$$
\mathcal{E}(\rho)=\sum_{i=1}^{\infty} M_{i} \rho M_{i}^{\dagger},
$$

where $\left\{M_{i}\right\} \subset \mathcal{B}(H, K)$ satisfies that $\sum_{i=1}^{\infty} M_{i}^{\dagger} M_{i}=I_{H}\left(\sum_{i=1}^{\infty} M_{i}^{\dagger} M_{i} \leq I_{H}\right)$.

\section{Elementary operator CRITERION OF SEPARABILITY}

Using the characterization of positive maps that are NCP in Section 2, we can get some criteria of entanglement of quantum states based on the positive map criterion. These will help us to deduce a necessary and sufficient criterion of separability of states.

The following necessary and sufficient condition for a state on finite dimensional spaces to be entangled is an immediate consequence of Corollary 2.7 and Horodeckis' Theorem.

Theorem 4.1. Let $H$ and $K$ be finite dimensional complex Hilbert spaces and $\rho$ be a state acting on $H \otimes K$. Then $\rho$ is an entangled state if and only if there exists a linear map of the form $\Phi(\cdot)=\sum_{i=1}^{k} C_{i}(\cdot) C_{i}^{\dagger}-\sum_{j=1}^{l} D_{j}(\cdot) D_{j}^{\dagger}: \mathcal{B}(H) \rightarrow \mathcal{B}(K)$ with $\left\{D_{1}, \ldots, D_{l}\right\}$ a contractive locally linear combination of $\left\{C_{1}, \ldots, C_{k}\right\}$, such that the operator $(\Phi \otimes I) \rho$ is not positive.

We will show below that this result is also true for infinite dimensional case. Before doing this, we write directly from Theorem 2.5 and Corollary 2.6 two sufficient criteria of entanglement of states for infinite dimensional systems.

Proposition 4.2. Let $H, K$ be complex Hilbert spaces and $\rho$ be a state on $H \otimes K$. Then $\rho$ is entangled if there exists an elementary operator of the form $\Phi(\cdot)=\sum_{i=1}^{k} C_{i}(\cdot) C_{i}^{\dagger}-$ $\sum_{j=1}^{l} D_{j}(\cdot) D_{j}^{\dagger}: \mathcal{B}(H) \rightarrow \mathcal{B}(K)$, where $\left\{D_{1}, \ldots, D_{l}\right\}$ is a contractive locally linear combination but not a contractive linear combination of $\left\{C_{1}, \ldots, C_{k}\right\}$, such that the operator $(\Phi \otimes I) \rho$ is not positive.

More generally, we have

Proposition 4.3. Let $H, K$ be complex Hilbert spaces and $\rho$ be a state on $H \otimes K$. Then $\rho$ is an entangled state if there exists a generalized elementary operator $\Phi$ defined by

$$
\Phi(X)=\sum_{i} A_{i} X A_{i}^{\dagger}-\sum_{j} C_{j} X C_{j}^{\dagger}
$$

for every $X \in \mathcal{B}(H)$, where $\left\|\sum_{i} A_{i} A_{i}^{\dagger}\right\|<\infty$ and $\left\|\sum_{j} C_{j} C_{j}^{\dagger}\right\|<\infty,\left\{C_{j}\right\}_{j}$ is a generalized contractive locally linear combination but not a generalized contractive linear combination of $\left\{A_{i}\right\}_{i}$, such that $(\Phi \otimes I) \rho$ is not positive. 
Propositions 4.2 and 4.3 only provide sufficient conditions for a state to be entangled and are not easily applied practically. In fact, these conditions are also necessary, and thus we obtain a necessary and sufficient criterion for entanglement which we will call the elementary operator criterion. Much better can be reached. Note that an elementary operator $\Phi$ is of finite rank if and only if there exist finite rank operators $A_{i}, B_{i}, i=1,2, \cdots, k$, such that $\Phi(X)=\sum_{i=1}^{k} A_{i} X B_{i}$ [37. We will prove that every entangled state can be detected by a positive elementary operator of finite rank.

Theorem 4.4. (Elementary operator criterion) Let $H, K$ be complex Hilbert spaces and $\rho$ be a state on $H \otimes K$. Then $\rho$ is entangled if and only if there exists an elementary operator of the form $\Phi(\cdot)=\sum_{i=1}^{k} C_{i}(\cdot) C_{i}^{\dagger}-\sum_{j=1}^{l} D_{j}(\cdot) D_{j}^{\dagger}: \mathcal{B}(H) \rightarrow \mathcal{B}(K)$, where all $C_{i} s$ and $D_{j}$ s are of finite rank and $\left\{D_{1}, \ldots, D_{l}\right\}$ is a contractive locally linear combination of $\left\{C_{1}, \ldots, C_{k}\right\}$, such that the operator $(\Phi \otimes I) \rho$ is not positive.

Proof. The "only if" part follows from Proposition 4.2. For "if" part, assume that the state $\rho$ is inseparable. Take any orthonormal bases $\{|i\rangle\}$ and $\{|j\rangle\}$ of $H$ and $K$, respectively. For any positive integers $s \leq \operatorname{dim} H$ and $t \leq \operatorname{dim} K$, denote $P_{s t}=P_{s} \otimes Q_{t}$, where $P_{s}$ and $Q_{t}$ are finite rank projections onto the subspaces $H_{s}$ and $K_{t}$ spanned by $\{|i\rangle\}_{i=0}^{s}$ and $\{|j\rangle\}_{j=0}^{t}$, respectively. Since $\rho$ is entangled, by [38, Theorem 2], there exists $(s, t)$ such that $\rho_{s t}=$ $\operatorname{Tr}\left(P_{s t} \rho P_{s t}\right)^{-1} P_{s t} \rho P_{s t}$ is entangled. Regarding $\rho_{s t}$ as a state on $H_{s} \otimes K_{t}$. As $\operatorname{dim}\left(H_{s} \otimes K_{t}\right)<$ $\infty$, by Theorem 4.1 , there exists a positive map $\Delta: \mathcal{B}\left(H_{s}\right) \rightarrow \mathcal{B}\left(K_{t}\right)$ of the form $\Delta(\cdot)=$ $\sum_{i=1}^{k} A_{i}(\cdot) A_{i}^{\dagger}-\sum_{j=1}^{l} B_{j}(\cdot) B_{j}^{\dagger}$ with $\left\{B_{1}, \ldots, B_{l}\right\}$ a contractive locally linear combination but not a contractive linear combination of $\left\{A_{1}, \ldots, A_{k}\right\}$, such that the operator $\left(\Delta \otimes Q_{t}\right) \rho_{s t}$ is not positive on $K_{t} \otimes K_{t}$. Let $\Phi: \mathcal{B}(H) \rightarrow \mathcal{B}(K)$ be defined by $\Phi(X)=Q_{t} \Delta\left(P_{s} X P_{s}\right) Q_{t}$. Then $\Phi$ is positive and $\Phi(X)=\sum_{i=1}^{k} C_{i}(X) C_{i}^{\dagger}-\sum_{j=1}^{l} D_{j}(X) D_{j}^{\dagger}$, where $C_{i}=Q_{t} A_{i} P_{s}$ and $D_{j}=Q_{t} B_{i} P_{s}$ are of finite rank.

Represent $\rho$ as an operator matrix $\rho=\left(\eta_{i j}\right)_{i, j}$ according to the bases $\{|i\rangle\}_{i=0}^{s}$ and $\{|j\rangle\}_{j=0}^{t}$, where $\eta_{i j} \in \mathcal{B}(H)$. Obviously,

$$
\rho_{s t}=\operatorname{Tr}\left(P_{s t} \rho P_{s t}\right)^{-1}\left(\begin{array}{cccc}
P_{s} \eta_{11} P_{s} & P_{s} \eta_{12} P_{s} & \cdots & P_{s} \eta_{1 t} P_{s} \\
P_{s} \eta_{21} P_{s} & P_{s} \eta_{22} P_{s} & \cdots & P_{s} \eta_{2 t} P_{s} \\
\vdots & \vdots & \ddots & \vdots \\
P_{s} \eta_{t 1} P_{s} & P_{s} \eta_{t 2} P_{s} & \cdots & P_{s} \eta_{t t} P_{s}
\end{array}\right)
$$

Thus we have

$$
\left(\Delta \otimes Q_{t}\right) \rho_{s t}=\operatorname{Tr}\left(P_{s t} \rho P_{s t}\right)^{-1}\left(\begin{array}{cccc}
\Delta\left(P_{s} \eta_{11} P_{s}\right) & \Delta\left(P_{s} \eta_{12} P_{s}\right) & \cdots & \Delta\left(P_{s} \eta_{1 t} P_{s}\right) \\
\Delta\left(P_{s} \eta_{21} P_{s}\right) & \Delta\left(P_{s} \eta_{22} P_{s}\right) & \cdots & \Delta\left(P_{s} \eta_{2 t} P_{s}\right) \\
\vdots & \vdots & \ddots & \vdots \\
\Delta\left(P_{s} \eta_{t 1} P_{s}\right) & \Delta\left(P_{s} \eta_{t 2} P_{s}\right) & \cdots & \Delta\left(P_{s} \eta_{t t} P_{s}\right)
\end{array}\right)
$$


is not positive. Note that $\Phi\left(\eta_{i j}\right)=Q_{t} \Delta\left(P_{s} \eta_{i j} P_{s}\right) Q_{t}=\Delta\left(P_{s} \eta_{i j} P_{s}\right)$. So

$$
=\left(\begin{array}{cccc|cc}
(\Phi \otimes I) \rho & & & & & \\
\Delta\left(P_{s} \eta_{11} P_{s}\right) & \Delta\left(P_{s} \eta_{12} P_{s}\right) & \cdots & \Delta\left(P_{s} \eta_{1 t} P_{s}\right) & \Delta\left(P_{s} \eta_{1(t+1)} P_{s}\right) & \cdots \\
\Delta\left(P_{s} \eta_{21} P_{s}\right) & \Delta\left(P_{s} \eta_{22} P_{s}\right) & \cdots & \Delta\left(P_{s} \eta_{2 t} P_{s}\right) & \Delta\left(P_{s} \eta_{2(t+1)} P_{s}\right) & \cdots \\
\vdots & \vdots & \ddots & \vdots & \vdots & \ddots \\
\Delta\left(P_{s} \eta_{t 1} P_{s}\right) & \Delta\left(P_{s} \eta_{t 2} P_{s}\right) & \cdots & \Delta\left(P_{s} \eta_{t t} P_{s}\right) & \Delta\left(P_{s} \eta_{t(t+1)} P_{s}\right) & \cdots \\
\hline \Delta\left(P_{s} \eta_{(t+1) 1} P_{s}\right) & \Delta\left(P_{s} \eta_{(t+1) 2} P_{s}\right) & \cdots & \Delta\left(P_{s} \eta_{(t+1) t} P_{s}\right) & \Delta\left(P_{s} \eta_{(t+1)(t+1)} P_{s}\right) & \cdots \\
\vdots & \vdots & \ddots & \vdots & \vdots & \ddots
\end{array}\right) .
$$

It follows that $(\Phi \otimes I) \rho$ is not positive since it has a non positive $t \times t$ submatrix (4.1). The proof is completed.

To sum up, we have proved the following criterion of separability, which is valid for both finite and infinite dimensional systems, improves Stømer's theorem [22] and is easier to practise by our characterization of positive elementary operators.

Theorem 4.5. (Elementary operator criterion) Let $H, K$ be complex Hilbert spaces and $\rho$ be a state acting on $H \otimes K$. Then the following statements are equivalent.

(1) $\rho$ is separable;

(2) $(\Phi \otimes I) \rho \geq 0$ holds for every positive elementary operator $\Phi: \mathcal{B}(H) \rightarrow \mathcal{B}(K)$.

(3) $(\Phi \otimes I) \rho \geq 0$ holds for every finite-rank positive elementary operator $\Phi: \mathcal{B}(H) \rightarrow \mathcal{B}(K)$.

\section{Examples of NCP positive maps and Entangled states}

It follows from Theorem 4.4, 4.5 and Theorem 2.11, for both finite and infinite dimensional systems, it is very important to construct NCP positive linear maps between matrix algebras since the non-complete positivity of a positive elementary operator is essentially determined by its behavior on finite-dimensional subspaces. In this section we give some concrete examples of NCP positive linear maps between matrix algebras by applying the results in Section 2, and, according to the elementary operator criterion, some of them are used to recognize entangled states that cannot be recognized by the PPT criterion and the realignment criterion.

Let $H$ be a complex Hilbert space of $\operatorname{dim} H=n<\infty$ and let $\{|1\rangle,|2\rangle, \ldots,|n\rangle\}$ be an orthonormal basis of $H$. Denote $E_{i j}=|i\rangle\langle j|, 1 \leq i, j \leq n$. The well known NCP positive map on $\mathcal{B}(H)$, that is, the transpose $T \mapsto T^{t}$ is an elementary operator

$$
T^{t}=\sum_{i=1}^{n} E_{i i} T E_{i i}+\sum_{i<j} A_{i j} T A_{i j}^{\dagger}-\sum_{i<j} C_{i j} T C_{i j}^{\dagger} \quad \forall T,
$$

where $A_{i j}=\frac{1}{\sqrt{2}}\left(E_{i j}+E_{j i}\right), C_{i j}=\frac{1}{\sqrt{2}}\left(E_{i j}-E_{j i}\right)$. Another example of well known NCP positive map is the reduction map, which has the form

$$
T \mapsto \operatorname{Tr}(T) I-T=\sum_{i \neq j} E_{i j} T E_{j i}+\sum_{i \neq j} G_{i j} A G_{i j}^{\dagger}-\sum_{i \neq j} F_{i j} A F_{i j}^{\dagger} \quad \forall T,
$$

where $F_{i j}=\frac{1}{\sqrt{2}}\left(E_{i i}+E_{j j}\right)$ and $G_{i j}=\frac{1}{\sqrt{2}}\left(E_{i i}-E_{j j}\right)$.

Next we give another kind of NCP positive linear maps.

Proposition 5.1. Let $H$ be a complex Hilbert space of $2 \leq \operatorname{dim} H=n<\infty$ and let $\{|1\rangle,|2\rangle, \ldots,|n\rangle\}$ be an orthonormal basis of $H$. Denote $E_{i j}=|i\rangle\langle j|, 1 \leq i, j \leq n$. Let 
$A_{k}=\sum_{i=1}^{n} a_{k i}|i\rangle\langle i|, k=1, \ldots, s$ and $B_{l}=\sum_{i=1} b_{l i}|i\rangle\langle i|, l=1, \ldots, t$ with $t>0$ and $s+t \leq n$. Assume that $\left\{A_{k}, B_{l}: k=1, \ldots, s ; l=1, \ldots, t\right\}$ is a linearly independent set. Let $\Delta: \mathcal{B}(H) \rightarrow$ $\mathcal{B}(H)$ be the linear map defined by

$$
\Delta(T)=\sum_{k=1}^{s} A_{k} T A_{k}^{\dagger}+\sum_{i \neq j} E_{i j} T E_{i j}^{\dagger}-\sum_{l=1}^{t} B_{l} T B_{l}^{\dagger}
$$

for every $T \in \mathcal{B}(H)$. If $\sum_{k=1}^{s}\left|a_{k i}\right|^{2} \geq \sum_{l=1}^{t}\left|b_{l i}\right|^{2},\left|\sum_{k=1}^{s} a_{k i} a_{k j}-\sum_{l=1}^{s} b_{l i} b_{l j}\right| \leq 1$ whenever $i \neq j$, then $\Delta$ is NCP positive.

Proof. It is clear that $\Delta$ defined in Eq.(5.1) is not completely positive since $B_{j}$ is linearly independent to $\left\{A_{k}, E_{i j}: 1 \leq k \leq s ; 1 \leq i, j \leq n, i \neq j\right\}$. Assume that $\sum_{k=1}^{s}\left|a_{k i}\right|^{2} \geq$ $\sum_{l=1}^{t}\left|b_{l i}\right|^{2},\left|\sum_{k=1}^{s} a_{k i} a_{k j}-\sum_{l=1}^{s} b_{l i} b_{l j}\right| \leq 1$ whenever $i \neq j$, We will show that $\Delta$ is positive.

Note that

$$
\Delta\left(E_{m m}\right)=\left(\sum_{k=1}^{s}\left|a_{k m}\right|^{2}-\sum_{l=1}^{t}\left|b_{l m}\right|^{2}\right) E_{k k}+\sum_{i \neq k} E_{i i}
$$

and

$$
\Delta\left(E_{i j}\right)=\left(\sum_{k=1}^{s} a_{k i} \bar{a}_{k j}-\sum_{l=1}^{t} b_{l i} \bar{b}_{l j}\right) E_{i j} \quad \text { if } i \neq j .
$$

Let $f_{i i}=\sum_{k=1}^{s}\left|a_{k i}\right|^{2}-\sum_{l=1}^{t}\left|b_{l i}\right|^{2}$ and $f_{i j}=\sum_{k=1}^{s} a_{k i} \bar{a}_{k j}-\sum_{l=1}^{t} b_{l i} \bar{b}_{l j}$ if $i \neq j$. Clearly, $f_{j i}=\bar{f}_{i j}$ for all $i, j$.

Identify $H$ with $\mathbb{C}^{n}$. For any $|\psi\rangle=\left(\xi_{1}, \xi_{2}, \ldots, \xi_{n}\right)^{T} \in \mathbb{C}^{n}$, consider the rank-one positive matrix $|\psi\rangle\langle\psi|=\left(\xi_{i} \bar{\xi}_{j}\right.$ ). By Eqs.(5.2) and (5.3) we have

$$
\begin{aligned}
& \Delta(|\psi\rangle\langle\psi|)=\left(\begin{array}{cccc}
f_{11}\left|\xi_{1}\right|^{2} & f_{12} \xi_{1} \bar{\xi}_{2} & \cdots & f_{1 n} \xi_{1} \overline{\xi_{n}} \\
f_{21} \xi_{2} \bar{\xi}_{1} & f_{22}\left|\xi_{2}\right|^{2} & \cdots & f_{2 n} \xi_{2} \bar{\xi}_{n} \\
\vdots & \vdots & \ddots & \vdots \\
f_{n 1} \xi_{n} \bar{\xi}_{1} & f_{n 2} \xi_{n} \bar{\xi}_{2} & \cdots & f_{n n}\left|\xi_{n}\right|^{2}
\end{array}\right) \\
& \begin{aligned}
& +\left(\begin{array}{cccc}
\sum_{1 \leq j \leq n, j \neq 1}\left|\xi_{j}\right|^{2} & 0 & \cdots & 0 \\
0 & \sum_{1 \leq j \leq n, j \neq 2}\left|\xi_{j}\right|^{2} & \cdots & 0 \\
\vdots & \vdots & \ddots & \vdots \\
0 & 0 & \cdots & \sum_{1 \leq j \leq n, j \neq n}\left|\xi_{j}\right|^{2}
\end{array}\right) \\
\geq & \left(\begin{array}{cccc}
\sum_{1 \leq j \leq n, j \neq 1}\left|\xi_{j}\right|^{2} & f_{12} \xi_{1} \bar{\xi}_{2} & \cdots & f_{1 n} \bar{\xi}_{1} \bar{\xi}_{n} \\
f_{21} \xi_{2} \bar{\xi}_{1} & \sum_{1 \leq j \leq n, j \neq 2}\left|\xi_{j}\right|^{2} & \cdots & f_{2 n} \xi_{2} \bar{\xi}_{n} \\
\vdots & \vdots & \ddots & \vdots \\
f_{n 1} \xi_{n} \bar{\xi}_{1} & f_{n 2} \xi_{n} \bar{\xi}_{2} & \cdots & \sum_{1 \leq j \leq n, j \neq n}\left|\xi_{j}\right|^{2}
\end{array}\right) \\
= & C_{\psi}
\end{aligned}
\end{aligned}
$$

So it suffices to show that $C_{\psi} \geq 0$.

To do this, denote $c_{i}=\left|\xi_{i}\right|$. Then, by the assumption of $\left|f_{i j}\right| \leq 1$ for $i \neq j$, we have $f_{i j} \xi_{i} \bar{\xi}_{j}=c_{i} c_{j} v_{i j}$ with $\left|v_{i j}\right| \leq 1$, and

$$
C_{\psi}=\left(\begin{array}{cccc}
\sum_{1 \leq j \leq n, j \neq 1} c_{j}^{2} & c_{1} c_{2} v_{12} & \cdots & c_{1} c_{n} v_{1 n} \\
c_{1} c_{2} v_{12}^{-} & \sum_{1 \leq j \leq n, j \neq 2} c_{j}^{2} & \cdots & c_{2} c_{n} v_{2 n} \\
\vdots & \vdots & \ddots & \vdots \\
c_{1} c_{n} v_{1 n}^{-} & c_{2} c_{n} v_{2 n}^{-} & \cdots & \sum_{1 \leq j \leq n, j \neq n} c_{j}^{2}
\end{array}\right) .
$$


For any $|\phi\rangle=\left(\eta_{1}, \eta_{2}, \ldots, \eta_{n}\right)^{T} \in \mathbb{C}^{n}$, writing $d_{i}=\left|\eta_{i}\right|$, we have

$$
\begin{aligned}
\left\langle\phi \mid C_{\psi} \phi\right\rangle & =\sum_{i=1}^{n}\left(\sum_{1 \leq j \leq n, j \neq i} c_{j}^{2}\right)\left|\eta_{i}\right|^{2}+2 \operatorname{Re}\left(\sum_{i<j} c_{i} c_{j} v_{i j} \eta_{j} \bar{\eta}_{i}\right) \\
& \geq \sum_{i=1}^{n}\left(\sum_{1 \leq j \leq n, j \neq i} c_{j}^{2}\right) d_{i}^{2}-2 \sum_{i<j} c_{i} c_{j} d_{i} d_{j} \\
& =\sum_{i<j}\left(c_{i} d_{j}-c_{j} d_{i}\right)^{2} \geq 0 .
\end{aligned}
$$

Therefore, $C_{\psi} \geq 0$. We have proved that $\Delta(|\psi\rangle\langle\psi|) \geq 0$ holds for all rank-one positive matrices $|\psi\rangle\langle\psi|$. It follows that $\Delta$ is a positive linear map, as desired.

The next result gives a NCP positive maps on $3 \times 3$ matrices.

Proposition 5.2. Let $\Gamma: M_{3}(\mathbb{C}) \rightarrow M_{3}(\mathbb{C})$ be defined by

$$
\begin{aligned}
\Gamma(A)= & \sum_{i=1}^{3} E_{i i} A E_{i i}+E_{12} A E_{21}+E_{23} A E_{32}+E_{31} A E_{13} \\
& +\sum_{i \neq j} G_{i j} A G_{i j}^{\dagger}-\sum_{i \neq j} F_{i j} A F_{i j}^{\dagger}
\end{aligned}
$$

for all $A$, where $F_{i j}=\frac{1}{2}\left(E_{i i}+E_{j j}\right)$ and $G_{i j}=\frac{1}{2}\left(E_{i i}-E_{j j}\right), i, j=1,2,3$ and $i \neq j$. Then $\Gamma$ is positive and indecomposable.

It is clear that $\Gamma$ is not completely positive by the results in Section 2 . We will show that $\Gamma$ is positive. There is a easy way to check it. Obviously, $\Gamma$ maps $A=\left(a_{i j}\right)$ to the matrix

$$
\Gamma(A)=\left(\begin{array}{ccc}
a_{11}+a_{22} & -a_{12} & -a_{13} \\
-a_{21} & a_{22}+a_{33} & -a_{23} \\
-a_{31} & -a_{32} & a_{33}+a_{11}
\end{array}\right)
$$

So, we need only check that, if

$$
D=\left(\begin{array}{lll}
a & c & f \\
\bar{c} & b & e \\
\bar{f} & \bar{e} & d
\end{array}\right)
$$

is positive, then

$$
\tilde{D}=\left(\begin{array}{ccc}
a+b & -c & -f \\
-\bar{c} & b+d & -e \\
-\bar{f} & -\bar{e} & d+a
\end{array}\right)
$$

is positive, and this suffices to show that $\operatorname{det}(\tilde{D}) \geq 0$. As $D \geq 0$, we have

$$
a b d+c e \bar{f}+\bar{c} \bar{e} f-b|f|^{2}-a|e|^{2}-d|c|^{2} \geq 0 .
$$

Also, there are numbers $t, s, r \in \mathbb{C}$ with $|t| \leq 1,|s| \leq 1$ and $|r| \leq 1$ such that $c=\sqrt{a b}$, $e=\sqrt{b d} s$ and $f=\sqrt{a d} r$. Thus

$$
\operatorname{det}(\tilde{D}) \geq a^{2} d+a b^{2}+b d^{2}+a b d \geq 0
$$

The map $\Gamma$ is also an example that is indecomposable. Recall that a positive elementary operator decomposable if it has the form $\Delta_{1}+\Delta_{2}^{t}$ for some completely positive elementary 
operators $\Delta_{1}$ and $\Delta_{2}$. Now, for any positive numbers $a, b$ with $a b \geq 1$, let $\rho \in \mathcal{S}\left(\mathbb{C}^{3} \otimes \mathbb{C}^{3}\right)$ be

$$
\rho=\frac{1}{3(1+a+b)}\left(\begin{array}{ccccccccc}
1 & 0 & 0 & 0 & 1 & 0 & 0 & 0 & 1 \\
0 & a & 0 & 1 & 0 & 0 & 0 & 0 & 0 \\
0 & 0 & b & 0 & 0 & 0 & 1 & 0 & 0 \\
0 & 1 & 0 & b & 0 & 0 & 0 & 0 & 0 \\
1 & 0 & 0 & 0 & 1 & 0 & 0 & 0 & 1 \\
0 & 0 & 0 & 0 & 0 & a & 0 & 1 & 0 \\
0 & 0 & 1 & 0 & 0 & 0 & a & 0 & 0 \\
0 & 0 & 0 & 0 & 0 & 1 & 0 & b & 0 \\
1 & 0 & 0 & 0 & 1 & 0 & 0 & 0 & 1
\end{array}\right)=\frac{1}{3(1+a+b)} \rho_{0}
$$

where $a \neq 1$. $\rho$ is a PPT state since $\rho$ is symmetric under partial transpose. However,

$$
\left(I_{3} \otimes \Gamma\right)\left(\rho_{0}\right)=\left(\begin{array}{ccccccccc}
1+a & 0 & 0 & 0 & -1 & 0 & 0 & 0 & -1 \\
0 & a+b & 0 & -1 & 0 & 0 & 0 & 0 & 0 \\
0 & 0 & b+1 & 0 & 0 & 0 & -1 & 0 & 0 \\
0 & -1 & 0 & b+1 & 0 & 0 & 0 & 0 & 0 \\
-1 & 0 & 0 & 0 & 1+a & 0 & 0 & 0 & -1 \\
0 & 0 & 0 & 0 & 0 & a+b & 0 & -1 & 0 \\
0 & 0 & -1 & 0 & 0 & 0 & a+b & 0 & 0 \\
0 & 0 & 0 & 0 & 0 & -1 & 0 & b+1 & 0 \\
-1 & 0 & 0 & 0 & -1 & 0 & 0 & 0 & 1+a
\end{array}\right)
$$

is not positive. Indeed, the vector $(1,0,0,0,1,0,0,0,1)^{t}$ is an eigenvector of $\left(I_{3} \otimes \Gamma\right)\left(\rho_{0}\right)$ with eigenvalue $a-1$, which is negative for $a<1$. This ensures the inseparability of the PPT state $\rho$. Consequently, $\Gamma$ is indecomposable.

In the case $a>1$, inseparability of $\rho$ can't be detected by the map $\Gamma$. Let $\Gamma^{\prime}$ be a linear map defined by

$$
\begin{aligned}
\Gamma^{\prime}(A)= & \sum_{i=1}^{3} E_{i i} A E_{i i}+E_{21} A E_{12}+E_{32} A E_{23}+E_{13} A E_{31} \\
& +\sum_{i \neq j} G_{i j} A G_{i j}^{\dagger}-\sum_{i \neq j} F_{i j} A F_{i j}^{\dagger}
\end{aligned}
$$

for all $A$, where $F_{i j}=\frac{1}{2}\left(E_{i i}+E_{j j}\right)$ and $G_{i j}=\frac{1}{2}\left(E_{i i}-E_{j j}\right), i, j=1,2,3$ and $i \neq j . \Gamma^{\prime}$ is a positive map as well and it maps

$$
\left(\begin{array}{ccc}
a_{11} & a_{12} & a_{13} \\
a_{21} & a_{22} & a_{23} \\
a_{31} & a_{32} & a_{33}
\end{array}\right) \mapsto\left(\begin{array}{ccc}
a_{11}+a_{33} & -a_{12} & -a_{13} \\
-a_{21} & a_{22}+a_{11} & -a_{23} \\
-a_{31} & -a_{32} & a_{33}+a_{22}
\end{array}\right)
$$

By a simple calculation, we get that $\left(I_{3} \otimes \Gamma^{\prime}\right)\left(\rho_{0}\right)$ has a negative eigenvalue $b-1$ whenever $b<1$. Namely, $\rho$ is detected by the NCP positive map $\Gamma^{\prime}$.

Finally, we illustrate that the positive map $\Gamma$ (as well as $\Gamma^{\prime}$ ) can detect some entangled states that cannot be detected by the realignment criterion. To see this let 


$$
\rho_{1}=\frac{1}{195}\left(\begin{array}{ccccccccc}
0.99 & 0 & 0 & 0 & 0.99 & 0 & 0 & 0 & 0.99 \\
0 & 63 & 0 & 0 & 0 & 0 & 0 & 0 & 0 \\
0 & 0 & 1.01 & 1.01 & 0 & 0 & 0 & 1.01 & 0 \\
0 & 0 & 1.01 & 1.01 & 0 & 0 & 0 & 1.01 & 0 \\
0.99 & 0 & 0 & 0 & 0.99 & 0 & 0 & 0 & 0.99 \\
0 & 0 & 0 & 0 & 0 & 63 & 0 & 0 & 0 \\
0 & 0 & 0 & 0 & 0 & 0 & 63 & 0 & 0 \\
0 & 0 & 1.01 & 1.01 & 0 & 0 & 0 & 1.01 & 0 \\
0.99 & 0 & 0 & 0 & 0.99 & 0 & 0 & 0 & 0.99
\end{array}\right) .
$$

Then $\rho_{1}$ is a PPT state. The realignment matrix of $\rho_{1}$ is

$$
\rho_{1}^{R}=\frac{1}{195}\left(\begin{array}{ccccccccc}
0.99 & 0 & 0 & 0 & 63 & 0 & 0 & 0 & 1.01 \\
0 & 0.99 & 0 & 0 & 0 & 0 & 1.01 & 0 & 0 \\
0 & 0 & 0.99 & 0 & 0 & 0 & 0 & 1.01 & 0 \\
0 & 0 & 1.01 & 0.99 & 0 & 0 & 0 & 0 & 0 \\
1.01 & 0 & 0 & 0 & 0.99 & 0 & 0 & 0 & 63 \\
0 & 1.01 & 0 & 0 & 0 & 0.99 & 0 & 0 & 0 \\
0 & 0 & 0 & 0 & 0 & 1.01 & 0.99 & 0 & 0 \\
0 & 0 & 0 & 1.01 & 0 & 0 & 0 & 0.99 & 0 \\
63 & 0 & 0 & 0 & 1.01 & 0 & 0 & 0 & 0.99
\end{array}\right) .
$$

By computation, we have that the trace norm $\left\|\rho_{1}^{R}\right\|_{1} \doteq 0.9705<1$. Thus the realignment criterion does not apply to $\rho_{1}$. Note that,

$$
\begin{gathered}
\left(I_{3} \otimes \Gamma\right)\left(\rho_{1}\right) \\
=\frac{1}{195}\left(\begin{array}{ccccccccc}
63.99 & 0 & 0 & 0 & -0.99 & 0 & 0 & 0 & -0.99 \\
0 & 64.01 & 0 & 0 & 0 & 0 & 0 & 0 & 0 \\
0 & 0 & -1.01 & 2 & 0 & 0 & 0 & -1.01 & 0 \\
0 & 0 & -1.01 & 2 & 0 & 0 & 0 & -1.01 & 0 \\
-0.99 & 0 & 0 & 0 & 63.99 & 0 & 0 & 0 & -0.99 \\
0 & 0 & 0 & 0 & 0 & 64.01 & 0 & 0 & 0 \\
0 & 0 & 0 & 0 & 0 & 0 & 64.01 & 0 & 0 \\
0 & 0 & -1.01 & -1.01 & 0 & 0 & 0 & 2 & 0 \\
-0.99 & 0 & 0 & 0 & -0.99 & 0 & 0 & 0 & 63.99
\end{array}\right) .
\end{gathered}
$$

The eigenvalues of the last matrix are

$$
\left\{-\frac{2}{19500}, \frac{301}{19500}, \frac{301}{19500}, \frac{6401}{19500}, \frac{6401}{19500}, \frac{6401}{19500}, \frac{6201}{19500}, \frac{6498}{19500}, \frac{6498}{19500}\right\} .
$$

Thus $\left(I_{3} \otimes \Gamma\right)\left(\rho_{1}\right)$ has a negative eigenvalue and hence is not positive. This reveals that $\rho_{1}$ is entangled, however cannot be detected by the realignment criterion.

\section{Conclusion}

Let $H$ and $K$ be complex Hilbert spaces of any dimension. The well known positive map criterion of separability for finite dimensional quantum systems has been generalized to infinite dimensional quantum systems by Stømer recently which asserts that a state $\rho$ on 
$H \otimes K$ is separably if and only if $(\Phi \otimes I) \rho$ is positive for any normal positive linear maps $\Phi: \mathcal{B}(H) \rightarrow \mathcal{B}(K)$. However, this criterion is not practically applied because of the complicacy of normal positive linear maps. In this paper we give a concrete characterization of positive completely bounded normal linear maps by showing that a completely bounded normal linear map $\Phi$ is positive if and only if it has the form of $\Phi(X)=\sum_{i=1}^{\infty} A_{i} X A_{i}^{\dagger}-\sum_{j=1}^{\infty} C_{j} X C_{j}^{\dagger}$ for all $X$, where $\left\{C_{j}\right\}$ is a generalized contractive locally linear combination of $\left\{A_{i}\right\}$; furthermore, $\Phi$ is completely positive if and only if $\left\{C_{j}\right\}$ is a generalized contractive linear combination of $\left\{A_{i}\right\}$, and in turn, if and only if $\Phi$ has the form of $\Phi(X)=\sum_{i=1}^{\infty} B_{i} X B_{i}^{\dagger}$. This particularly gives a characterization of NCP positive elementary operators. Recall that a linear map $\Phi$ is called an elementary operator if it has the form of $\Phi(\cdot)=\sum_{i=1}^{n} A_{i}(\cdot) B_{i}$ with $n<\infty$. If both $H$ and $K$ are finite dimensional, all linear maps $\Phi: \mathcal{B}(H) \rightarrow \mathcal{B}(K)$ are elementary operators. Thus an elementary operator $\Phi$ is NCP positive if and only if there exist $C_{1}, \cdots, C_{k}, D_{1}, \cdots, D_{l} \in$ $\mathcal{B}(H, K)$ such that $\Phi(X)=\sum_{i=1}^{k} C_{i} X C_{i}^{\dagger}-\sum_{j=1}^{l} D_{j} X D_{j}^{\dagger}$ for all $X \in \mathcal{B}(H)$, and $\left\{D_{j}\right\}_{j=1}^{l}$ is a contractive locally linear combination but not a contractive linear combination of $\left\{C_{i}\right\}_{i=1}^{k}$. Therefore, for elementary operators, the question when positivity ensures complete positivity may be reduced to the question when contractive locally linear combination implies linear combination. This connection allows us to look more deeply into the relationship and the difference between positivity and complete positivity, and obtain some simple criteria to check whether a positive elementary operator is completely positive or not. This is important especially when we construct positive maps and apply them to recognize entanglement.

Above characterization of positive maps allows us to get a concrete representation of quantum channels for infinite dimensional systems that is similar to finite dimensional case. Every Quantum channel $\mathcal{E}$ has the form of $\mathcal{E}(\rho)=\sum_{i=1}^{\infty} M_{i} \rho M_{i}^{\dagger}$, where $\sum_{i=1}^{\infty} M_{i}^{\dagger} M_{i}=I$.

Much more importantly, our characterization of positive maps leads to a necessary and sufficient condition of separability which we call the elementary operator criterion: $\rho$ is separable if and only if $(\Phi \otimes I) \rho \geq 0$ holds for every positive elementary operator $\Phi: \mathcal{B}(H) \rightarrow \mathcal{B}(K)$, and in turn, if and only if $(\Phi \otimes I) \rho \geq 0$ holds for every finite-rank positive elementary operator $\Phi: \mathcal{B}(H) \rightarrow \mathcal{B}(K)$. Equivalently, $\rho$ is entangled if and only if there exists an elementary operator of the form $\Phi(\cdot)=\sum_{i=1}^{k} C_{i}(\cdot) C_{i}^{\dagger}-\sum_{j=1}^{l} D_{j}(\cdot) D_{j}^{\dagger}: \mathcal{B}(H) \rightarrow \mathcal{B}(K)$, where all $C_{i}$ s and $D_{j} \mathrm{~s}$ are of finite rank and $\left\{D_{1}, \ldots, D_{l}\right\}$ is a contractive locally linear combination of $\left\{C_{1}, \ldots, C_{k}\right\}$, such that the operator $(\Phi \otimes I) \rho$ is not positive. Obviously, this criterion is more practical than the positive map criterion and the Stømer's theorem. Some examples of finite rank NCP positive elementary operators are given which are used to detect some entangled states that can not be recognized by the PPT criterion and the realignment criterion.

Acknowledgement. The authors wish to give their thanks to the referees for helpful comments and suggestions to improve the original manuscript.

\section{REFERENCES}

[1] Bengtsson I, Zyczkowski K, Cambridge University Press, Cambridge, 2006.

[2] Nielsen M A, Chuang I L, Quantum Computation and Quantum Information, Cambridge University Press, Cambridge, 2000.

[3] Werner R F, Phys. Rev. A 40 (1989) 4277.

[4] Bennett C H, Brassard G, Crépeau C, Jozsa R, Peres A, Wootters W K, Phys. Rev. Lett. 70 (1993) 1895.

[5] Bouwmeester D, Pan J W, Mattle K, Eibl M, Weinfurter H, Zeilinger A, Nature 390 (1997) 575. 
[6] Deutsch D, Ekert A, Jozsa R, Macchiavello C, Popescu S, Sanpera A, Phys. Rev. Lett. 77 (1996) 2818.

[7] Deutsch D, Ekert A, Jozsa R, Macchiavello C, Popescu S, A. Sanpera, Phys. Rev. Lett. 80 (1998) 2022.

[8] Shor P W, Phys. Rev. A 52 (1995) 2493.

[9] Horodecki M, Horodecki P, Horodecki R, Phys. Lett. A, 223 (1996), 1-8.

[10] Peres A, Phys. Lett. A 202 (1996) 16.

[11] Horodecki M, Horodecki P, Horodecki R, Phys. Lett. A 80 (1998) 5239.

[12] Chen K, Wu L A, Quant. Inf. Comput 3 (2003) 193.

[13] Horodecki M, Horodecki P, Phys. Rev. A, 59 (1999), 4206.

[14] Cerf N J, Adami C, Gingrich R M, Phys. Rev. A 60 (1999): 893.

[15] Horodecki R, Horodecki P, Horodecki M, Rev. Mod. Phys. 81 (2009) 865.

[16] Hou J C, Qi X F, Phys. Rev. A 81 (2010) 062351.

[17] Salgado D, Sánchez-Gómez J L, Open Systems and Information Dynamics, 12(1) (2005), 55-64.

[18] Skowronek E, Størmer E, Życzkowski K, J. Math. Phys. 50, 062106 (2009); doi:10.1063/1.3155378

[19] Grabowski J, Kuś M, Marmo G, Open. Sys. and Information Dyn. 14 (2007), 355-370.

[20] Kye S-H, Trends in Mathematics, Information Center for Mathematical Sciences, 6(2) (2003), 83-91.

[21] Kadison R V, Ringrose J R, Fundamentals of the Theorey of Operator Algebras II, Graduate Studies in Math., 16, American Math. Society, New York: Academic Press, 1983.

[22] Størmer E, J. Func. Anal., 254 (2008), 2304-2313.

[23] Dixmier J, Von Neumann Algebras, North-Holland Publishing Com., Amsterdan, New York, Oxford, 1981.

[24] Chruściński D, Kossakowski A, Open Systems and Inf. Dynamics 14 (2007) 275;

[25] Chruściński D, Kossakowski A, J. Phys. A: Math. Theor. 41 (2008) 145301.

[26] Augusiak R, Stasinska J, Phys. Rev. A 77 (2008) 010303.

[27] Choi M D, Lin. Alg. Appl., 10(1975), 285-290.

[28] Choi M D, J. Operator Theory, 4(1980), 271-285.

[29] Choi M D, Proc. Sympos. Pure Math., 38(1982), 583-590.

[30] Depillis J, Pacific J. Math., 23 (1967), 129-137.

[31] Hou J C, J. Operator Theory, 39 (1998), 43-58.

[32] Paulsen V, Completely Bounded Maps and Operator Algebras, Cambridge Studies in Advanced Mathematics 78, Cambridge University Press, Cambridge, 2002.

[33] Stinespring W F, Proc. Amer. Math. Soc., 6 (1955), 211-216.

[34] Hou J C, Sci. in China (ser.A), 32(1989), 929-940.

[35] Mathieu M, Math. Ann., 284(1989), 223-244.

[36] Effros E G, Ruan Z-J, Operator Spaces, Clarendon Press, Oxford, 2000.

[37] Hou J C, Sci. in China (ser.A), 36(9) (1993), 1025-1035.

[38] Sperling J, Vogel W, Phys. Rev. A 79 (2009) 052313.

Department of Mathematics, Taiyuan University of Technology, Taiyuan 030024, P. R. Of China

E-mail address: jinchuanhou@yahoo.com.cn, houjinchuan@tyut.edu.cn 